\title{
Multi-Hop Communication is Order-Optimal for Homogeneous Sensor Networks
}

\author{
Arnab Chakrabarti \\ Department of Electrical and \\ Computer Engineering \\ Rice University, MS-366 \\ 6100 Main Street \\ Houston, Texas - 77005 \\ arnychak@rice.edu
}

\author{
Ashutosh Sabharwal \\ Department of Electrical and \\ Computer Engineering \\ Rice University, MS-366 \\ 6100 Main Street \\ Houston, Texas - 77005 \\ ashu@rice.edu
}

\author{
Behnaam Aazhang \\ Department of Electrical and \\ Computer Engineering \\ Rice University, MS-366 \\ 6100 Main Street \\ Houston, Texas - 77005 \\ aaz@rice.edu
}

\begin{abstract}
The main goal of this paper is to show that multi-hop singleuser communication achieves the per node transport capacity of $\Theta\left(\frac{\ln N}{N}\right)$ in homogeneous sensor networks, making it order-optimal. Our contributions in this paper are threefold. First, we construct a route-discovery and scheduling scheme based on spatial TDMA for sensor networks. Second, we show that our schedule achieves a per node transport capacity of $\Theta\left(\frac{\ln N}{N}\right)$, the same as that achievable by beamforming. Third, we compare multi-hop communication and beamforming based methods in terms of the network power consumption required to attain a fixed throughput. Based on our power calculations, we conclude that if the channel attenuation is above a certain threshold (which we calculate), then multi-hop communication performs better, whereas below the threshold, beamforming is preferable.
\end{abstract}

\section{Categories and Subject Descriptors}

H.1.1 [Models and Principles]: Systems and Information Theory-Information theory; C.2.2 [Computer Communication Networks]: Network management-Network Protocols

\section{General Terms}

performance of communication schemes, design of schedule in multi-hop sensor network

\section{Keywords}

sensor network, communication, power, throughput, transport capacity, multi-hop, beamforming, schedule, TDMA

Permission to make digital or hard copies of all or part of this work for personal or classroom use is granted without fee provided that copies are not made or distributed for profit or commercial advantage and that copies bear this notice and the full citation on the first page. To copy otherwise, to republish, to post on servers or to redistribute to lists, requires prior specific permission and/or a fee.

IPSN'04, April 26-27, 2004, Berkeley, California, USA.

Copyright 2004 ACM 1-58113-846-6/04/0004 ...\$5.00.

\section{INTRODUCTION}

Communication schemes for sensor networks commonly utilize node cooperation to improve throughput or to save power. Most of these cooperative communication methods fall into one of two main categories. ${ }^{1}$ The first category consists of communication schemes that rely on symbol level cooperation between nodes. Many examples exist in the literature, such as the relay channel [5], the interference channel $[2,4]$, and many recent works $[11,8,17,6,13,18,12$, 16]. The second category includes schemes that are built on contention avoidance, and where the data traverses the network using multiple hops [14]. Multi-hop communication does not exploit any diversity, performs no interference cancellation, and is perhaps the only operational mode of communication in existing sensor networks.

Although the capacity of the first category of cooperative communication is almost always higher than the second, the practicality of building actual sensor networks begs the question: how suboptimal are sensor networks that use multi-hop communication? With network throughput as the metric of performance, we focus on how capacity scales with addition of more nodes in a fixed area. Our main result is that multi-hop communication achieves the best possible order of growth in network throughput $\Theta\left(\frac{\ln N}{N}\right)$, making it orderoptimal.

To show order optimality of multi-hop communication, we construct a simple network schedule which always achieves the network throughput of $L / 3$, where $L$ is the maximum per-link throughput. Combined with appropriate choice of communication range, the proposed schedule leads to an order-optimal network. The proposed schedule is not optimal for all topologies, and higher network throughputs are possible in many cases. The schedule might be of independent interest by itself, since existence of such a schedule was proven in [14], but no explicit construction was provided.

Having demonstrated the order optimality of multi-hop communication, we compare the power consumption of two constructive schemes which are known to have the same capacity scaling behavior: multi-hop communication proposed in this paper, and beamforming [6]. For the case when the total network power is constrained, we show that multi-hop

\footnotetext{
${ }^{1}$ Mobility can improve capacity, if used appropriately in conjunction with schemes belonging to either of these two categories $[9,3,21]$. However, discussion of mobility is out of the scope of this paper.
} 
communication outperforms the beamforming based methods for all channels with pathloss exponent $\gamma>2$ in a dense network. Refining the metric to look at the maximum power consumed by any node in the network, we find that multihop communication outperforms beamforming for pathloss exponents greater than $1+\sqrt{5} \approx 3.23$. The advantage of beamforming is that all nodes consume equal power. In contrast, multi-hop communication suffers from the fact that a few nodes near the observer carry significantly more data than peripheral nodes, leading to disparity in node power consumptions.

Note that a multi-hop network was also analyzed in [14], where it was shown to achieve an order of $\Theta\left(\frac{1}{N}\right)$. Our proposed scheme achieves a better order primarily because we choose the range of communication appropriately, to take advantage of the fact that a denser network remains connected with a smaller range, leading to power savings.

The rest of this paper is organized as follows. In Section 2, we describe our model for a sensor network. This is followed by Section 3, which provides an overview of multi-hop and beamforming schemes for communication in a sensor network. After the preliminaries, we describe a self-organizing routing and scheduling scheme in Section 4, which is shown to achieve the transport capacity in sensor networks in Section 5. Section 6 provides a power comparison of multi-hop and beamforming schemes based on average network power as well as worst case node power. Finally, we conclude in Section 7 with comments on the relative merits of different sensor network communication schemes and a discussion of open questions.

\section{SENSOR NETWORK MODEL}

In this section, we describe our sensor network model. A sensor network consists of $N$ static nodes distributed in a uniformly IID fashion over a planar disc of unit area. The network has a single centrally placed observer (also called sink or destination). Sensors collect data, and communicate it to the observer. The network is homogeneous i.e., the nodes are all identical, and they collect data at the same rate. The network, as a whole, collects data at a fixed rate $D$. Sensor nodes are each equipped with a single omnidirectional antenna, and we assume that all communication takes place over gaussian channels with bandwidth $B$. The channel is characterized by an attenuation constant $\gamma$, which describes how the signal power falls with distance. If the transmitted power ${ }^{2}$ is $P$, then the received power at a distance $d$ is $\frac{P}{d^{\gamma}}$. We further assume that the channels are time-invariant.

The network has access to limited energy resources. We model this limitation in two different ways. For a target network longevity, the energy restriction translates into a restriction on the average network power. Therefore, we impose the constraint that the average network power must not exceed $P_{a v g}$.

Restricting the average network power is useful for measuring the efficacy of a communication scheme. However, it fails to capture the fact that each node has its own power source. Individual power sources limit the power available to individual nodes, which is more restrictive than a power

\footnotetext{
${ }^{2}$ Throughout this paper, by power we mean power normalized with noise. In other words, the noise power $B N_{0}$ is normalized to unity.
}

limitation for the entire network. In a geographically distributed sensor network, nodes may consume very different amounts of communication power, and consequently, some nodes may run out of power earlier than others. Thus, the lifetime of the node that consumes the most power (and expires first) is an indicator of network lifetime. Keeping this in mind, we also compare communication schemes for networks in which power per node is constrained.

We also assume that the data collected at different nodes is independent. Such an assumption is not valid in general. However, our treatment can be extended to situations where there is spatial correlation, by making use of distributed coding strategies for correlated sources [20].

We look closely at two communication schemes for sensor networks: multi-hop single-user communication, and beamforming based communication. Both of these schemes are described in Section 3.

\section{COMMUNICATION SCHEMES}

Although several different communication schemes can be used in sensor networks, the following two - multi-hop and beamforming - are emerging as prominent ones, as evidenced in literature $[15,14,6,16]$. Direct communication between individual sensors and the observer is another option that has been considered by researchers. However, in our model, direct communication will consume significantly more power than both beamforming and multi-hop. This is because direct communication neither exploits the beamforming gain, nor benefits from the reduced range between neighboring sensors in a dense network. Therefore, we do not elaborate on direct communication here.

\subsection{Multi-hop Communication}

In multi-hop communication [14], every sensor has the same range $r$, and may communicate or interfere with other sensors or with the observer, if and only if they are within range. The average power consumption of the network is bounded by $P_{a v g}$. Nodes communicate at a fixed data rate $L$ (link capacity for a range $r$ ), which does not depend on the distance between sensors as long as they are within range. The same power is used in every single hop transmission. In other words, sensors do not perform power control. Sensors far from the observer send data to it by relaying data through a chain of sensor-to-sensor links. Communication between any two nodes is of the single user type. No other form of node cooperation is permitted.

It has been shown in [14] that the capacity of a multi-hop sensor network is $\Theta\left(\frac{1}{N}\right)$ assuming that the link capacity is not a function of $N$. We will show in Section 5 that for a power constrained sensor network, the capacity is $\Theta\left(\frac{\ln N}{N}\right)$, because the link capacity is related to $N$ through the communication range. This result holds even if the sensors do not use power control. To show this, in Section 4, we will first prove a few results that bound the achievable throughput for multi-hop communication, and then propose a routing and scheduling protocol that can achieve the proposed capacity of $\Theta\left(\frac{\ln N}{N}\right)$.

\subsection{Communication using Beamforming}

Here, we describe the strategy that was proposed in Section 3 of [6], extended to a 2-D scenario in a fairly obvious way. The average power consumption of the network is bounded by $P_{a v g}$. Communication between any sensor and 


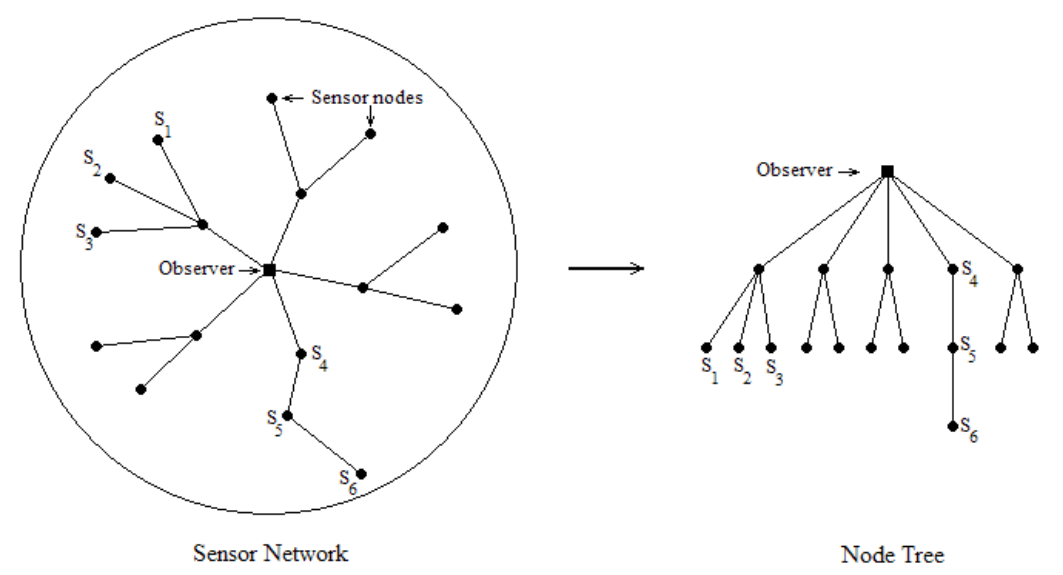

Figure 1: Multi-hop Sensor Tree

the observer takes place in two stages over two equal length time slots. In the first stage, the sensor (call it S1) transmits its information omnidirectionally with a power $P_{\text {avg }}$, and at a data rate which is $\alpha \ln N$ for some $\alpha>0$. In a dense sensor network, the data is successfully received in an area around $S 1$, which contains $N^{\alpha}$ nodes with high probability. In the second stage, all nodes that have received data from $S 1$, share $P_{a v g}$ equally, and use beamforming to send this data to the observer. Although, in the second stage, each sensor transmits at a power $\frac{P_{a v g}}{N^{\alpha}}$, the received power grows as $N^{\alpha} P_{a v g}$, due to the beamforming gain. Consequently, the throughput in the second stage is also $\approx \alpha \ln N$. Since the throughput achieved in both stages is $\Theta(\ln N)$, the net throughput is also $\Theta(\ln N)$. Therefore, the scheme achieves a per node transport capacity of $\Theta\left(\frac{\ln N}{N}\right)$. For further details of this scheme, and calculation of the constant $\alpha$, the reader is referred to [6].

\section{ROUTING AND SCHEDULING SCHEME FOR MULTI-HOP SENSOR NETWORKS}

The following is a brief outline of this section:

- First, we explain flooding, and show that in our framework, it can be used to obtain minimum-hop routes from every node to the observer.

- Next, we find bounds on network throughput. It is known from [14] that the network throughput is upper bounded by the link capacity $L$. We show, by a counterexample, that this upper bound is not achievable for some connected topologies.

- We further show that a network throughput of $\frac{L}{3}$ is achievable for all connected topologies.

- We present a simple schedule that can achieve a network throughput of $\frac{L}{3}$.

\subsection{Minimum-hop Route Discovery}

Since transmission power and data rates are identical for all links in our framework, the power consumed in sending information over a route is proportional to the number of hops, which makes fewest hop routes optimal. Fewest-hop routes between the observer and each of the sensors can be found by flooding the network starting from the observer.
After flooding, the nodes are organized into a tree with the observer at its root. A brief description of flooding follows.

\subsubsection{Flooding}

It is assumed that each node in the sensor network has a distinct address, which is assigned when sensors are manufactured. In the first step of flooding, the observer (root of the node tree) broadcasts a wake-up packet. Sensors within range of the observer receive this packet, and form the Level 1 nodes of the tree. These nodes, in turn, repeat the process of broadcasting, and discover new nodes, which are registered as Level 2 nodes. In this process, if packets from multiple nodes collide, they are retransmitted. The probability of collision among packets is reduced using random backoffs. Each Level 2 node has exactly one Level 1 node as its parent. If a node receives multiple wake-up packets, it responds to only the first one, and ignores all others. The process repeats until all nodes in the network have been found.

We note that flooding, as described above, requires time synchronization. Synchronization will also be necessary for the schedule we will propose in Section 4.4. A number of time synchronization protocols have been proposed and evaluated in the literature, see for example [7, 19]. Though no proof exists in the literature on the minimum overhead needed for maintaining desired accuracy of time synchronization, the current schemes seem to indicate that accurate synchronization can be achieved with fairly small amount of network's power and bandwidth resources. We realize that a more accurate analysis, which accounts for timing inaccuracies, is desirable but out of scope of this paper.

Any node can obtain a route to the observer by climbing up the node tree (see Figure 1). It is not hard to see that the routes discovered in this manner are minimum-hop routes if the channels are not time-varying (proved in Appendix A).

\subsection{Upper Bound May Not be Achievable}

Since communication is exclusively of the single-user type, the observer can receive data over no more than a single link at a time. Further, since the throughput of each link is $L$, the capacity of the entire network is upper bounded by $L$ [14].

Although it is easy to see that the link capacity $L$ is an upper bound on the network throughput, a network through- 
put $L$ may not be achievable because of interference ${ }^{3}$. When one node transmits, none of the other nodes, with which it interferes, can receive. In Figure 2, the consequence of interference is severe. Whenever any Level 1 sensor, let it be $S_{1}$, sends data to the observer, the remaining Level 1 sensors, $S_{2}$ and $S_{3}$, cannot receive data. Eventually, when $S_{1}$ runs out of data, the remaining sensors have almost no new data to send because they have not been receiving ${ }^{4}$. To resolve this, there must be periods during which none of the Level 1 nodes transmits; in other words, the observer receives nothing over some intervals. Therefore, the observer receives only through a single link and with intermittent periods of no reception, thereby demonstrating that the network throughput falls short of the link capacity in this situation.

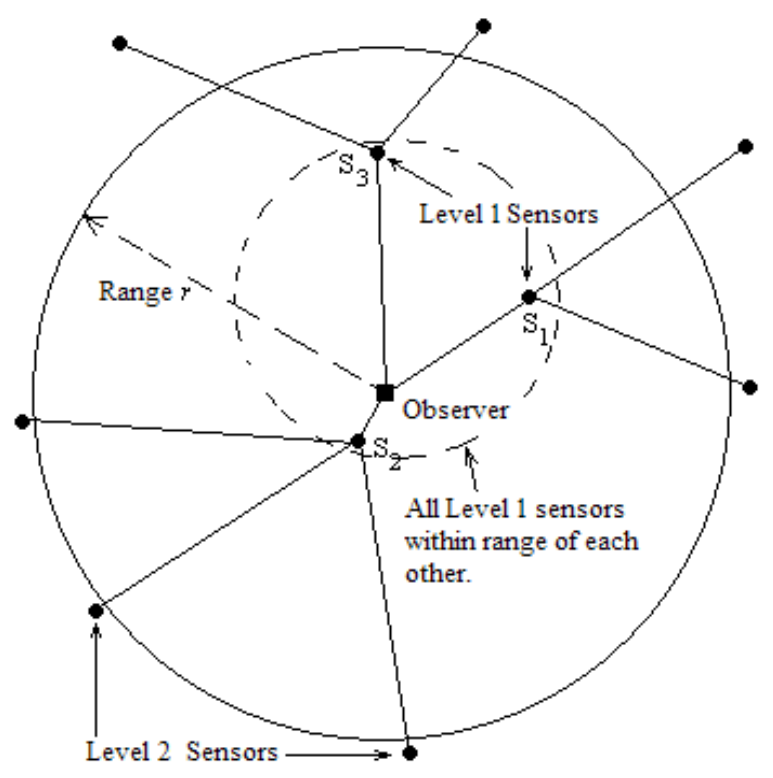

Sensor Network

Figure 2: A poor arrangement of nodes. All Level 1 nodes are within range of each other.

\subsection{Achievable Throughput}

Having shown by counterexample that a network throughput of $L$ is not always achievable, next we demonstrate that a link capacity of $\frac{L}{3}$ is always achievable. To prove this, we claim that a node $S_{i}$ in Level $i$ of the tree cannot interfere with another node $S_{j}$ unless $S_{j}$ belongs to one of the Levels $i-1, i$ or $i+1$ in the tree. This is easy to prove by contradiction. If $S_{i}$, belonging to Level $i$, interferes with $S_{j}$, belonging to Level $j$, where $|j-i|>1$, then connecting nodes $i$ and $j$ with a branch would bring one of them closer to the tree root by at least one hop, thereby contradicting the fact that the tree constructed by flooding is a

\footnotetext{
${ }^{3}$ In this discussion, nodes are said to interfere when they lie within range of each other but no branch exists between them in the tree formed by flooding (eg. nodes $S_{1}$ and $S_{2}$ in Figure 1).

${ }^{4}$ The remaining sensors will have a very small amount of data, which they have collected on their own. But since the link capacity is chosen to support data collection from the entire network, this data collected by a handful of sensors will be communicated in almost no time.
}

minimum-hop tree. As a result, when a node belonging to Level $i$ transmits, it does not prevent any node belonging to Level $j$ from receiving unless $j \in\{i-1, i, i+1\}$. Further, since a node belonging to Level $j$ receives data only from nodes belonging to Level $j+1$ (data flows towards the observer), this also means that a node in Level $i$ transmitting may not prevent another node in Level $j$ from transmitting unless $j \in\{i, i+1, i+2\}$. So, we see that single nodes in Levels $\ldots, i-3, i, i+3, \ldots$ can transmit simultaneously with no possibility of interference. If the link capacity is $L$, then consecutive levels can share time equally to avoid mutual interference and still ensure that data flows towards the observer at an average rate of $\frac{L}{3}$ (see Figure 3 ).

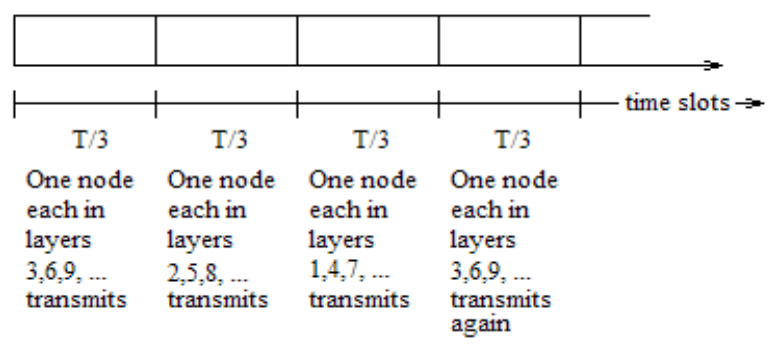

Figure 3: Time Sharing among different levels

\subsection{A Time-Sharing Schedule}

Our argument for proving that an average throughput of $\frac{L}{3}$ is achievable using a link capacity of $L$, suggests a simple scheduling scheme based on time-sharing. We shall show in Section 5.2 that this schedule achieves a transport capacity of $\Theta\left(\frac{\ln N}{N}\right)$. The following are the salient features of this proposed scheme (see Figure 3):

- Time is divided into equal slots of length $T$.

- Slots are further divided in equal sub-slots of length $\frac{T}{3}$. The sub-slots are numbered consecutively $1,2,3$.

- Nodes belonging to layers $3,6,9, \ldots$ transmit during sub-slot 1. Nodes belonging to layers $2,5,8, \ldots$ transmit during sub-slot 2. Nodes belonging to layers $1,4,7, \ldots$ transmit during sub-slot 3 .

- Within each sub-slot, multiple nodes may transmit in any sequence, but two or more nodes may not transmit simultaneously.

Some comments must be made regarding this schedule. First, a transport capacity greater than $\frac{L}{3}$ may be achievable by using a better schedule. But if nodes are randomly scattered, there may exist bad spatial node arrangements ${ }^{5}$ in which a link capacity of $L$ cannot ensure a transport capacity of more than $\frac{L}{3}$ (see Figure 4 ). So, in a sense, a network throughput of $\frac{L}{3}$ is all that can be guaranteed when node locations are not known a priori. Second, even when node locations are known, we expect the problem of finding an optimal schedule to be NP-hard. The NP-completeness of scheduling problems for packet radio networks is confirmed [1, 22]. This is likely to be true for sensor networks also.

\footnotetext{
${ }^{5}$ Such arrangements occur rarely in dense networks [14].
} 


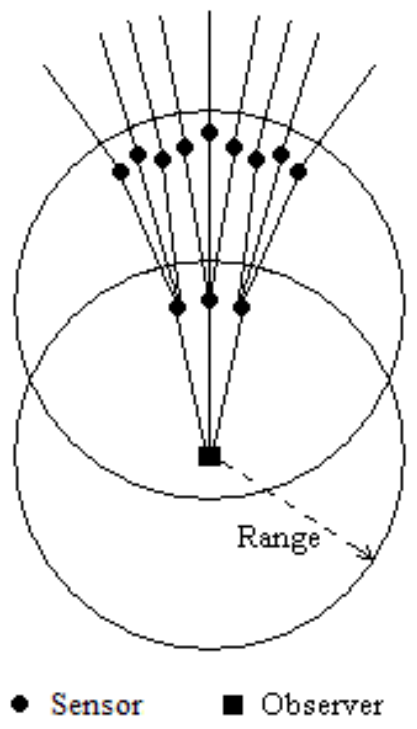

Figure 4: Worst Case Node Positions: Every node interferes with every other node in its own level as well as in the next higher and lower level.

\section{ORDER-OPTIMALITY OF MULTI-HOP COMMUNICATION}

We present our main result in the following theorem.

THEOREM 1. In a sensor network with a constraint on the average network transmit power, the transport capacity per node achievable by multi-hop single-user communication scales as $\Theta\left(\frac{\ln N}{N}\right)$, as the number of nodes $N$ in a fixed area grows large.

Proof. Our proof is in two parts. First, we present an upper bound on the order of the transport capacity, and then we show that the scheduling scheme of Section 4.4 achieves this order. Throughout, we work with the average network power, which is bounded by $P_{a v g}$.

\subsection{Upper Bound}

In [10], it was shown that in a network of $N$ nodes, if the range $r$ is written in terms of $N$ as

$$
\pi r^{2}=\frac{(\ln N+c(N))}{N},
$$

then the network is connected with unit probability if and only if

$$
\lim _{N \rightarrow \infty} c(N) \rightarrow \infty
$$

The above result serves as a useful guideline for choosing the transmission range in dense 2-D wireless networks.

Note from Equation 2 that the growth of $c(N)$ to $\infty$ can be arbitrarily slow. If $c(N)$ were chosen to be $\ln \ln N$, then for large $N, r \approx \sqrt{\frac{\ln N}{\pi N}}$. Therefore, we choose the range of communication to be $r=\sqrt{\frac{\ln N}{\pi N}}$. This slight inaccuracy will not alter any of the results presented in the paper ${ }^{6}$.

${ }^{6}$ Our choice of $r$ is further justified as follows. If we choose $c(N)=\ln N$ (which clearly satisfies Equation 2), then we
We now establish an upper bound on the link capacity, subject to the above network connectivity constraint. The power available to a single node clearly cannot exceed the total network power $P_{\text {avg }}$. Even if the node communicating directly with the observer were to consume all of the network power $P_{a v g}$, it would only achieve a throughput of

$$
L_{\text {upper }}=B \ln \left(1+\frac{P_{a v g}}{r^{\gamma}}\right)=B \ln \left(1+\frac{P_{a v g}}{\left(\frac{\ln N}{\pi N}\right)^{\frac{\gamma}{2}}}\right)
$$

where $B$ is the system bandwidth. For large $N$,

$$
\begin{aligned}
L_{\text {upper }} & =B \ln \left(1+\frac{P_{\text {avg }}}{\left(\frac{\ln N}{\pi N}\right)^{\frac{\gamma}{2}}}\right) \\
& \approx B \ln \left(\frac{P_{\text {avg }}}{\left(\frac{\ln N}{\pi N}\right)^{\frac{\gamma}{2}}}\right) \\
& =c \ln N-c^{\prime} \ln \ln N+c^{\prime \prime} \\
& \sim \Theta(\ln N) .
\end{aligned}
$$

where $c, c^{\prime}$, and $c^{\prime \prime}$ are constants and $c>0$. To sum up:

$$
\begin{aligned}
\text { Network Throughput } & \leq \text { Link Capacity } \\
& \leq L_{\text {upper }} \\
& \sim \Theta(\ln N) .
\end{aligned}
$$

Dividing by $N$, we conclude that the upper bound on the per node transport capacity is $\Theta\left(\frac{\ln N}{N}\right)$.

Note that this upper bound cannot be improved by using beamforming (see [6]).

\subsection{Achievable Capacity}

We will show now that a transport capacity of $\Theta\left(\frac{\ln N}{N}\right)$ is achieved using the schedule presented in Section 4.4. According to our schedule, at every instant of time, only one node transmits from every triplet of consecutive levels of the node tree. In a dense network of unit area, where the range of each node is $r=\sqrt{\frac{\ln N}{\pi N}}$, the number of levels in the tree will be approximately equal to the network radius divided by the range, which is $\frac{1 / \sqrt{\pi}}{\sqrt{\frac{\ln N}{\pi N}}}$. If, at any time, one node from every three levels transmits, and these nodes share the power $P_{a v g}$ equally, then the transmit power per node would be given by

$$
P_{t x}=3\left(\sqrt{\frac{\ln N}{N}}\right) P_{a v g}
$$

from which we calculate that the achievable link throughput is:

$$
\begin{aligned}
L_{\text {achievable }} & =B \ln \left(1+\frac{P_{t x}}{r^{\gamma}}\right) \\
& =B \ln \left(1+\frac{3 P_{a v g}}{\left(\frac{1}{\pi}\right)^{\frac{\gamma}{2}}\left(\frac{\ln N}{N}\right)^{\frac{\gamma-1}{2}}}\right)
\end{aligned}
$$

have $r=\sqrt{\frac{2 \ln N}{\pi N}}$. The reader may verify that our main conclusions remain same irrespective of whether we use $r=\sqrt{\frac{\ln N}{\pi N}}$ or $r=\sqrt{\frac{2 \ln N}{\pi N}}$ in the remainder of this paper. 
where $B$ is the system bandwidth. For large $N$,

$$
\begin{aligned}
L_{\text {achievable }} & =B \ln \left(1+\frac{3 P_{a v g}}{\left(\frac{1}{\pi}\right)^{\frac{\gamma}{2}}\left(\frac{\ln N}{N}\right)^{\frac{\gamma-1}{2}}}\right) \\
& \approx B \ln \left(\frac{3 P_{a v g}}{\left(\frac{1}{\pi}\right)^{\frac{\gamma}{2}}\left(\frac{\ln N}{N}\right)^{\frac{\gamma-1}{2}}}\right) \\
& =c \ln N-c^{\prime} \ln \ln N+c^{\prime \prime} \\
& \sim \Theta(\ln N)
\end{aligned}
$$

where $c, c^{\prime}$, and $c^{\prime \prime}$ are constants with $c>0$ if $\gamma>1$. It is not hard to see that $L_{\text {achievable }}$ is $\Theta(\ln N)$, since for practical wireless channels, $\gamma \geq 2$. Dividing by the number of nodes, we conclude that the achievable throughput per node is also $\Theta\left(\frac{\ln N}{N}\right)$. Hence the transport capacity is $\Theta\left(\frac{\ln N}{N}\right)$. This completes our proof.

\section{POWER COMPARISON OF MULTI-HOP AND BEAMFORMING}

In this section, our goal is to find out which communication scheme maximizes network longevity and under what set of conditions. Towards that end, we perform a comparison of the two communication schemes on the basis of average network power and worst case node power.

In order to compare the power consumptions of the two communication schemes in a fair manner, we equate their throughputs. Both networks are assumed to be collecting data at a fixed rate $D$, with each node collecting data at a rate $\frac{D}{N}$.

As stated in Section 2, we assume that the network is a unit area disc with the observer at its center. Earlier, it was mentioned that the sensor distribution is uniformly IID, which means that there may be more sensors in some areas and less in others. However, as the number of sensors becomes large, such imbalances become extremely improbable, as proved in lemma 1 of [14]. Hence, in our calculations of average power, we assume that the number of nodes in any area within the network is given by the area times the node density.

\subsection{Comparison on the Basis of Average Net- work Power}

\subsubsection{Average Network Power in a Multi-hop Sensor Network}

As shown in Section 4.3, guaranteeing a network throughput of $D$ may require a link capacity of up to $3 D$. Therefore, we calculate the average node power with a link capacity of $3 D$. The communication range in a dense network is $\sqrt{\frac{\ln N}{\pi N}}$, from considerations of network connectivity [10]. Assuming that communication between nodes takes place over sufficiently long time slots $T$ so as to invoke the capacity theorem for AWGN channels, the power per hop is given as

$$
P_{h o p}=\left(\frac{\ln N}{\pi N}\right)^{\frac{\gamma}{2}}\left(e^{\frac{3 D}{B}}-1\right) .
$$

In a time slot of length $T$, a single node collects $\frac{D T}{N}$ bits. With a link capacity of $3 D$, communication over any link lasts $\frac{T}{3 N}$ seconds.

The number of hops from a node at distance $d$ from the observer is lower bounded by $\left\lceil\frac{d}{\sqrt{\frac{\ln N}{\pi N}}}\right\rceil$. As $N$ becomes large, the number of hops becomes nearly equal to $\frac{d}{\sqrt{\frac{\ln N}{\pi N}}}$. From this, the expected number of hops from a randomly picked node to the observer is found to be

$$
E\left[N_{\text {hops }}\right]=\frac{2}{3} \sqrt{\frac{N}{\ln N}}
$$

Therefore, the total energy consumption of the network in a period $T$ is found as the product of the power per hop, the communication time per hop, the expected number of hops, and the total number of nodes in the network. Dividing by $T$ gives us the average network power, which is

$$
P_{\text {multihop }}=\frac{2}{9}\left(\frac{1}{\pi}\right)^{\frac{\gamma}{2}}\left(e^{\frac{3 D}{B}}-1\right)\left(\frac{\ln N}{N}\right)^{\frac{(\gamma-1)}{2}}
$$

\subsubsection{Average Network Power using Beamforming}

Communication between any node and the observer takes place in two consecutive stages over two equal length time slots. The transmission rate in each slot is chosen as $2 D$ so that an average rate of $D$ is achieved. In the first slot, the node transmits omnidirectionally at a power $P$, which is the average network power. This transmission is successfully received by other sensors within a range $r$ such that

$$
2 D=B \ln \left(1+\frac{P}{(r)^{\gamma}}\right)
$$

In the second slot, these $N \pi r^{2}$ nodes then perform beamforming to transmit this information to the observer. The same power $P$ is shared by all these nodes equally, from which we obtain

$$
2 D=B \ln \left(1+\frac{\pi r^{2} N P}{\left(\frac{1}{\sqrt{\pi}}\right)^{\gamma}}\right)
$$

where $\frac{1}{\sqrt{\pi}}$ is the radius of the network and therefore is the maximum distance of any node from the observer. By choosing this as range, we ensure that every node successfully communicates with the observer. From Equation 12 and Equation 13, we find that

$$
r=\frac{1}{\sqrt{\pi}(N)^{\frac{1}{(\gamma+2)}}}
$$

Since, in this mode of communication, the individual node power $P$ while communicating is the same as the average network power Pbeamforming, from Equation 12 and Equation 14, the network power is found as

$$
P_{\text {beamforming }}=\left(\frac{1}{\pi}\right)^{\frac{\gamma}{2}}\left(e^{\frac{2 D}{B}}-1\right)\left(\frac{1}{N}\right)^{\left(\frac{\gamma}{\gamma+2}\right)}
$$

In the above treatment, we have assumed that nodes at different distances from the observer use the same power in the second stage of communication, when they perform beamforming. But even if individual nodes were to perform power control to take advantage of their nearness to the observer, the network power would go down by a constant factor of $\frac{2}{2+\left(\frac{\gamma^{2}}{\gamma+2}\right)}$ only and this would have no bearing on the asymptotic behavior as $N \rightarrow \infty$.

\subsubsection{Power Comparison}

It is easy to see that as $N$ becomes large,

$$
\frac{P_{\text {multihop }}}{P_{\text {beamforming }}} \propto \frac{(\ln N)^{\frac{(\gamma-1)}{2}}}{(N)^{\frac{(\gamma-2)(\gamma+1)}{2(\gamma+2)}}}
$$


From the above relation, we can draw an interesting conclusion: that the relative power consumptions of the two schemes is solely dependent on the channel attenuation in a dense network. In particular, the network power necessary to ensure any finite network throughput $D$ using multi-hop communication is less than that using beamforming for all $\gamma>2$ if the network is dense. Since $\gamma \geq 2$ in all practical channels, multi-hop communication nearly always consumes less network power than communication using the beamforming strategy.

The function of $N$ in Equation 16 that governs the ratio of network power consumptions, is plotted in Figure 5 for five different values of $\gamma$. The plot shows that the trends predicted for large $N$ are valid in small networks $(N \geq 10)$ also.

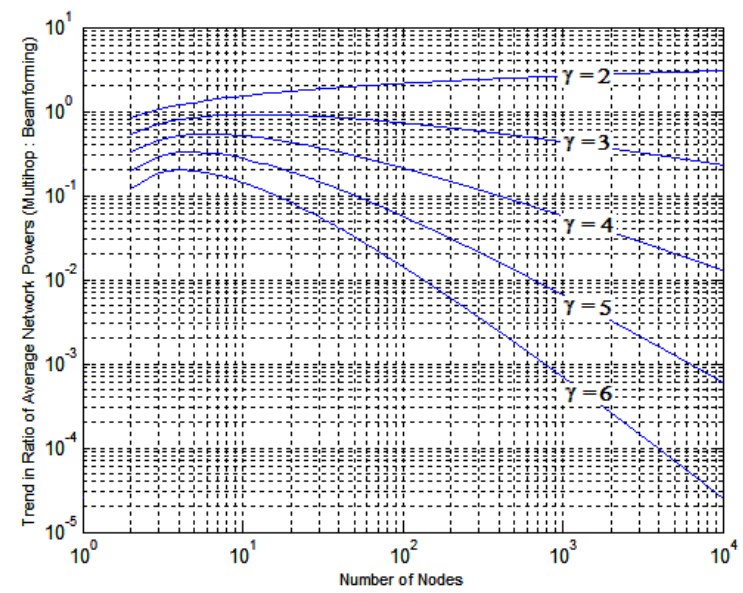

Figure 5: Trend in the Ratio of Average Network Powers of Beamforming vs. Multi-hop.

\subsection{Comparison on the Basis of Worst Node Power}

Here, we compare the schemes based on the worst node power. In the beamforming scheme described in [6], the average power consumption of all nodes is identical, which is a very desirable property. In a homogeneous network, all nodes are equipped with the same kind of power sources, and one node cannot transfer its power to another. In such a situation, if some nodes consume more power than others, they expire earlier than others, thereby impairing the network's ability to collect data. This is one of the major problems with multi-hop communication. Nodes that are near the observer carry much more data than nodes near the periphery of the network. The ratio of the time during which a peripheral node transmits to the period during which a node near the observer transmits can be a small as $\frac{1}{N}$ in the worst case. This is also the ratio of their powers if a time-shared scheduling scheme like the one proposed by us is used. Therefore, some nodes may be consuming $N$ times the power consumed by other nodes.

In the case of beamforming, the worst case average node power is the same as the average node power, which equals $\frac{P_{\text {beamforming }}}{N}$. In the case of multi-hop communication, the worst case scenario occurs when a single node within range of the observer carries all the data of the network. Again, assuming a data rate of $3 D$, we have

$$
P_{\text {worst-multihop }}=\frac{1}{3}\left(e^{\frac{3 D}{B}}-1\right)\left(\frac{\ln N}{\pi N}\right)^{\frac{\gamma}{2}} .
$$

The factor of $\frac{1}{3}$ accounts for the fact that at a rate of $3 D$, the node transmits only a third of the time. Thus we see that

$$
\frac{P_{\text {worst-multihop }}}{P_{\text {worst-beamforming }}} \propto \frac{(\ln N)^{\frac{\gamma}{2}}}{(N)^{\frac{\left(\gamma^{2}-2 \gamma-4\right)}{2(\gamma+2)}}}
$$

The above function of $N$, that governs the ratio of worst case node powers, is plotted in Figure 6 for different values of $\gamma$. In this case, we see that the asymptotic trend dominates only when $N$ is large $(\geq 1000)$ or when the channel is either very good or very poor $(\gamma \geq 5$ or $\gamma \leq 3)$.

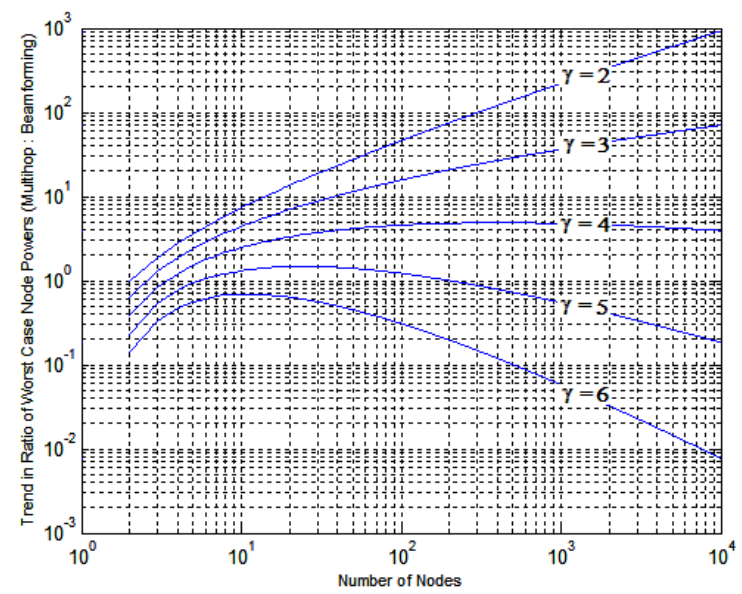

Figure 6: Trend in the Ratio of Worst Node Powers of Beamforming vs. Multi-hop.

Surprisingly, it is seen from Equation 18 that whenever $\gamma>1+\sqrt{5}=3.236$, multi-hop communication is still better for increasing network longevity, despite the disparities in node power consumption. However, we also see that when the channel attenuation is low, such as in small area networks where a direct line of sight path to the observer is available, beamforming is a better alternative.

\section{CONCLUDING REMARKS}

The simplest form of communication in a sensor network is perhaps direct communication between individual sensors and the observer. However, such communication is power inefficient and affords a low throughput. Both of the schemes discussed in this paper - multi-hop and beamforming - improve the network performance in different ways. Schemes based on beamforming improve the capacity by exploiting the fact that data can be shared with $N^{\alpha}$ neighboring nodes $(\alpha>0)$ at very little cost and these nodes can act as a multiple-transmit-antenna array to achieve a net throughput of $\Theta(\ln N)$. In multi-hop communication, the gain comes through a reduction in communication range leading to power reduction. Each of these schemes performs better than the other in a certain type of channel. If the channel attenuation with distance is high, it is profitable to use multi-hop because the power savings due to reduced 
range more than offset the beamforming gain. The opposite holds true when the channel attenuation is low.

Several important questions arise in the context of our work. The first is whether a combination of symbol-level cooperation and multi-hop communication can perform better than both of these schemes. We believe that the answer this question is in the affirmative (in terms of power). However, it is unlikely that such a scheme would achieve a transport capacity higher than $\Theta\left(\frac{\ln N}{N}\right)$. Second, the scheduling scheme proposed by us may achieve only a fraction of what an optimal schedule achieves. But finding an optimal schedule may be computationally infeasible. Thus, the question arises whether it is possible to find a schedule that is close to optimal with low complexity. We suspect that methods used for scheduling in packet radio networks should be useful for finding such schedules in sensor networks also. We further conjecture that in a dense sensor network, the maximum node power can be reduced by a factor of $\approx \ln N$ by using an appropriate schedule. Lastly, a combination of smart scheduling, and power control can further reduce network power, an option that needs to be explored.

\section{REFERENCES}

[1] E. Arikan. Some complexity results about packet radio networks. IEEE Trans. Info. Th., 30:681-685, July 1984.

[2] R. Benzel. The capacity region of a class of discrete additive degraded interference channels. IEEE Trans. Info. Th., 25(2):228-231, March 1979.

[3] A. Chakrabarti, A. Sabharwal, and B. Aazhang. Using predictable observer mobility for power efficient design of sensor networks. In IPSN 2003, pages 129-145, April 2003.

[4] M. H. M. Costa. On the Gaussian interference channel. IEEE Trans. Info. Th., 31(5):607-615, September 1985.

[5] T. M. Cover and A. A. El Gamal. Capacity theorems for the relay channel. IEEE Trans. Info. Th., 25:572-584, September 1979.

[6] H. El Gamal. On the scaling laws of dense wireless sensor networks. IEEE Trans. Info. Th., April 2003. submitted.

[7] J. Elson and D. Estrin. Time synchronization for wireless sensor networks. In Parallel and Distributed Processing Symposium, pages 1965-1970, April 2001.

[8] M. Gastpar and M. Vetterli. On the capacity of wireless networks: The relay case. In IEEE INFOCOM., June 2002.

[9] M. Grossglauser and D. Tse. Mobility increases the capacity of ad hoc wireless networks. In IEEE INFOCOM., volume 3, pages 1360-1369, 2001.

[10] P. Gupta and P. R. Kumar. Critical power for asymptotic connectivity in wireless networks. In Stochastic Analysis, Control, Optimization and Applications, Boston., pages 1106-1110, 1998.

[11] P. Gupta and P. R. Kumar. Towards an information theory of large networks: An achievable rate region. IEEE Trans. Info. Th., 49:1877-1894, August 2003.

[12] M. A. Khojastepour, A. Sabharwal, and B. Aazhang. Bounds on achievable rates for general multi-terminal networks with practical constraints. In Lecture Notes in Computer Science - 2634, pages 146-161, 2003.
[13] J. N. Laneman and G. W. Wornell. Energy-efficient antenna sharing and relaying for wireless networks. In Wireless Communications and Networking Conference, volume 1, pages 7-12, September 2000.

[14] D. Marco, E. J. Duarte-Melo, M. Liu, and D. L. Neuhoff. On the many-to-one transport capacity of a dense wireless sensor network and the compressibility of its data. In IPSN 2003, pages 1-16, April 2003.

[15] R. Min and A. Chandrakasan. Energy-efficient communication for ad-hoc wireless sensor networks. In Conference Record of the Thirty-Fifth Asilomar Conference on Signals, Systems and Computers, volume 1, pages 139-143, 2001.

[16] K. J. Quirk, M. Srinivasan, and J. R. Agre. Cooperative modulation techniques for long haul relay in sensor networks. In GLOBECOM, volume 2, pages 1171-1175, November 2001.

[17] B. Schein and R. Gallager. The gaussian parallel relay network. In IEEE ISIT 2000, page 22, June 2000.

[18] A. Sendonaris, E. Erkip, and B. Aazhang. Increasing uplink capacity via user cooperation diversity. In IEEE ISIT 1998, page 156, August 1998.

[19] M. Sichitiu and C. Veerarittiphan. Simple, accurate time synchronization for wireless sensor networks. In Wireless Communications and Networking, pages 1266-1273, March 2003.

[20] D. Slepian and J. Wolf. Noiseless coding of correlated information sources. IEEE Trans. Info. Th., 19:471-480, July 1973.

[21] E. Uysal-Biyikoglu and A. Keshavarzian. Throughput achievable with no relaying in a mobile interference network. In IEEE ISCC, pages 641-645, June 2003.

[22] G. Wang and N. Ansari. Optimal broadcast scheduling in packet radio networks using mean field annealing. IEEE Journal on Selected Areas in Communications, 15:250 -260, February 1997.

\section{APPENDIX}

\section{A. ROUTES DISCOVERED BY FLOODING ARE MINIMUM-HOP ROUTES}

TheOREM 2. A node registered as a Level n node through flooding (as described in Section 4.1.1) cannot have a path of length less than $n$ connecting it to the observer.

Proof. The proof bases itself on the following claim. Claim: If a node $N_{1}$ is within range of another node $N_{2}$, then the two nodes either belong to the same layer or they belong to consecutive layers in the tree formed by flooding. Proof of claim: Assume, without loss of generality, that $N_{1}$ is discovered first in the process of flooding and registered as a Level $k$ node. If $N_{2}$ is discovered in the same level, there is nothing to prove. Otherwise, since all new nodes that are within range of the Level $k$ nodes are registered as Level $k+1$ nodes, $N_{2}$ will be registered as a Level $k+1$ node, which proves the above claim.

Once this claim has been proved, the proof, which uses contradiction, is trivial. It is equivalent to proving that if $x_{i} \in\{-1,0,1\}$ then $\sum_{i=1}^{m} x_{i}$ cannot equal $n$ for any $m<$ $n$. 